\title{
Acceso al medicamento y derecho a la protección de la salud: régimen jurídico de los medicamentos huérfanos en la Unión Europea
}

Access to medication and right to health protection: the orphan drugs legal framework in the European Union

\section{Francisco Miguel Bombillar Sáenz}

Profesor de Derecho Administrativo. Universidad de Granada. España.

Resumen: Aunque el acceso al medicamento es un componente crucial dentro del contenido propio del derecho a la protección de la salud, millones de ciudadanos tienen, aún hoy, problemas para acceder a medicamentos esenciales para su salud, en los países en vías de desarrollo y en la Unión Europea. Se expone el régimen jurídico europeo que afecta a los medicamentos huérfanos, las políticas públicas emprendidas en España para, dentro del marco comunitario, atender a las necesidades de las personas afectadas por enfermedades raras, así como, por último, los muchos retos que aún quedan por resolver para conseguir garantizar a este colectivo el derecho a la protección de la salud en paridad de condiciones con el resto de ciudadanos.

Palabras clave: Unión Europea; derecho a la salud; medicamentos huérfanos; enfermedades raras.

Resumo: Ainda que o acesso aos medicamentos seja um componente essencial do Direito em relação à proteção da saúde, milhões de cidadãos ainda hoje encontram problemas para conseguir os medicamentos essenciais para a sua saúde, nos países em desenvolvimento e na União Europeia. Discute-se o regime jurídico europeu relativo aos medicamentos órfãos, as políticas públicas implementadas na Espanha para, dentro do quadro comunitário, atender as necessidades das pessoas afetadas pelas doenças raras bem como, por último, os muitos desafios que ainda precisam ser resolvidos para poder garantir a essa população esse direito coletivo da proteção da saúde com igualdade de condições com o resto dos cidadãos.

Palavras chaves: União Europeia; direito à saúde; medicamentos órfãos; doenças raras.

Abstract: Although access to medicine is a crucial component in the actual context of the right to health protection, millions of people still have trouble accessing essential medicines for health in developing countries and the European Union. Is exposed the European legal regime on orphan drugs, the public policies implemented in Spain within the EU framework, addressing the needs of people affected by rare diseases and, finally, the many challenges that remain to be solved to achieve to guarantee to this collective the right to health protection at parity basis with other citizens.

Key words: European Union; right to health protection; orphan drugs; rare diseases. 


\section{Propósito de este estudio y estructura argumentativa del mismo.}

El propósito de este artículo es analizar el régimen jurídico de los medicamentos huérfanos en la Unión Europea, prestando una particular atención a las políticas públicas emprendidas a este respecto por el Estado español, partiendo de la premisa de que el acceso a este tipo de medicamentos es uno de los pilares fundamentales sobre los que se asienta el derecho a la protección de la salud de las personas afectadas por enfermedades raras.

Para abordar este objeto de estudio, marcado por la premisa expuesta, analizamos aquí, a la luz del Derecho de la Unión Europea, el concepto jurídico de medicamento huérfano y de enfermedad rara, el procedimiento de designación como medicamento huérfano, el procedimiento centralizado de autorización de comercialización de este tipo de fármacos y su posibilidad de autorización condicional, junto a otras medidas de estímulo de cara a la concesión o mantenimiento de dicha autorización de comercialización (destacando el derecho de exclusividad comercial por diez años). Expuesto el marco comunitario de referencia, repasamos a continuación algunas de las políticas públicas puestas en marcha en el Estado español a favor de los afectados por enfermedades raras. Para finalizar, una vez descrito el régimen jurídico de los medicamentos huérfanos en la Unión Europea, apuntamos algunos de los problemas que quedan aún por resolver en materia de acceso al medicamento huérfano y nos atrevemos a individualizar algunas posibles líneas de actuación a tomar en consideración de cara al futuro.

\section{Premisa de partida: el acceso al medicamento como parte integrante del derecho a la protección de la salud.}

Los medicamentos son bienes económicos a través de los que se hace efectivo el derecho a la protección de la salud, consagrado en el artículo 43 de la Constitución Española, pues contribuyen a prevenir, curar o aliviar enfermedades y a corregir o reparar las secuelas provocadas por éstas.

Gracias a los medicamentos, la humanidad ha podido luchar eficazmente contra las enfermedades que, desde siempre, la han amenazado. Constituyen, en suma, un producto que contribuye a proporcionar una mejor calidad de vida y representan, al mismo tiempo, una esperanza para el futuro. Por todo ello, el acceso al medicamento se identifica inevitablemente como un componente crucial dentro del 
contenido propio del derecho a la protección de la salud (Barranco Vela y Bombillar Sáenz, 2010).

De acuerdo con la Conferencia Mundial sobre el uso racional de los medicamentos, celebrada en Nairobi en 1985, los pacientes han de recibir los medicamentos apropiados a sus necesidades clínicas, en las dosis adecuadas, en el momento oportuno y con el menor coste tanto para ellos como para la comunidad. El acceso a los medicamentos no se agota con el acto material de suministrarlos (Bergel, 2005), este acceso ha de reunir una serie de requisitos. El paciente ha de acceder al medicamento en el momento oportuno; un medicamento que ha de ser de calidad, suministrarse en las cantidades adecuadas para responder al tratamiento y, por supuesto, ser efectivo para el uso al que se le destina.

Al acceder a este fármaco, el paciente ha de ser capaz de sufragar su costo sin ver afectadas significativamente sus condiciones de vida, a la vez que ha de contar con una información adecuada sobre el mismo que le permita una utilización racional de este producto, en el marco de la tercera generación de leyes del medicamento.

La primera generación de leyes del medicamento exigió que los medicamentos fuesen seguros, pero no se ocupó de velar porque efectivamente éstos así lo fuesen. A partir de 1965, tras la tragedia de la Talidomida, en Europa podemos hablar de una segunda generación de leyes del medicamento. Desde ese momento, y buscando salvaguardar la salud pública de la ciudadanía, la Administración se ha ocupado de evaluar y controlar cada uno de los medicamentos que son puestos en el mercado (Bombillar Sáenz, 2010a y 2013b, entre otras publicaciones).

Calidad, seguridad y eficacia son los objetivos que guían el procedimiento de autorización de un fármaco. La Administración controla que los medicamentos (1) alcancen los requisitos de calidad establecidos; (2) que sean seguros, no produciendo en condiciones normales de uso efectos tóxicos o indeseables desproporcionados al beneficio que procuran; (3) que sean eficaces en las indicaciones terapéuticas aprobadas; y (4) que estén correctamente identificados y acompañados de la información precisa para su utilización.

Una vez puestas en marcha, bajo el liderazgo de la propia Unión Europea (Valverde López, 2014), las herramientas necesarias para lograr estos objetivos, ha irrumpido con fuerza en las últimas décadas una tercera generación en la que -sin descuidar los requisitos de seguridad, calidad y eficacia - se va un paso más allá, 
buscando la utilización "racional" del fármaco: que la ciudadanía sólo consuma aquellos medicamentos que respondan a un equilibrio entre utilidad terapéutica y coste. Los Estados miembros se han marcado como una línea prioritaria de trabajo: la accesibilidad a un sistema de calidad gestionado de manera sostenible (Garrido Cuenca, 2013), de una manera viable económicamente. Este es el gran reto de la asistencia sanitaria pública en la Unión Europea.

Aunque es cierto que producir esta "mercancía", de alto nivel tecnológico, requiere realizar por parte de la industria un largo y costoso esfuerzo en investigación e innovación, también lo es que los recursos del Estado son limitados, por lo que el derecho a la salud, en el marco de la tercera generación de leyes del medicamento y más en el actual escenario de grave crisis económica que nos asola, se ha visto modulado por consideraciones económicas, reconfigurándose el marco jurídico sobre el que se asientan las prestaciones que conforman y dan sustento a este derecho.

Así, por ejemplo, se han visto excluidos del sistema público los extranjeros que no sean titulares de una autorización para residir en el territorio español o se ha instaurado el copago (repago) para los pensionistas en relación con la prestación farmacéutica. Estos y otros "recortes sanitarios" son estudiados, entre otros autores, por Cantero Martínez, 2014 y Garrido Cuenca, 2014.

En definitiva, el Estado no sólo busca preservar la salud de la ciudadanía también la de las arcas públicas, que deben hacer frente a muchas otras necesidades de su población. En un mercado tan complejo como el del medicamento, donde el que decide (el médico prescriptor) ni paga ni consume el medicamento; el que lo consume, ni lo paga (al menos íntegramente) ni decide; y el que lo paga (el Estado), ni lo consume ni decide; es lógico que se implementen una serie de mecanismos para actuar sobre la dimensión económica del medicamento, tanto sobre su precio (con la fijación del precio industrial y de los márgenes comerciales) como sobre su financiación (con la financiación selectiva, el copago y los precios de referencia); y se promueva el llamado uso racional de este producto o la introducción o el fomento del mercado de genéricos ${ }^{1}$ (como pone de relieve en España el articulado de la

\footnotetext{
${ }^{1}$ De acuerdo con el art. 10.2.b) de la Directiva 2001/83, en su actual redacción, se entiende por medicamento genérico: "todo medicamento que tenga la misma composición cualitativa y cuantitativa en sustancias activas y la misma forma farmacéutica, y cuya bioequivalencia con el medicamento de referencia haya sido demostrada por estudios adecuados de biodisponibilidad."

Esta definición se ve completada con una serie de presunciones, algunas de ellas bastante controvertidas: "Las diferentes sales, ésteres, éteres, isómeros, mezclas de isómeros, complejos o derivados de una sustancia activa se considerarán una misma sustancia activa, a menos que tengan
} 
LGURMPS, analizado por autores como Baes, 2010; Bombillar Sáenz, 2010; o Vida Fernández, 2007). Mientras que todos podemos compartir este diagnóstico, la descripción de este escenario, el problema surge a la hora de implementar el alcance y los efectos concretos de las medidas diseñadas para dar respuesta al mismo.

En este sentido, cada Estado miembro, y ya no la Unión Europea - mientras que la intervención de orden público es una competencia comunitarizada, la de servicio público (esto es, la que engloba la prestación farmacéutica) permanece en manos de cada unos de los veintiocho Estados que hoy conforman la Unión (Bombillar Sáenz, 2010a) -, ha obrado del modo que ha estimado más oportuno para alcanzar los objetivos reseñados.

Demos algunas notas en relación con el caso español. Ante la acuciante crisis económica en la que vivimos, el Estado español ha legislado con ahínco, a través del discutible instituto del decreto-ley, diversas y controvertidas medidas con las que ha pretendido aminorar y controlar nuestro elevado gasto público farmacéutico. Gran parte de ellas se recogen en el Real Decreto-Ley 16/2012, de 20 de abril, de medidas urgentes para garantizar la sostenibilidad del Sistema Nacional de Salud (Larios Risco, Palomar Olmeda y Vázquez Garranzo, 2012).

Teóricamente, estos mecanismos de fijación o de control de precios, así como los procedimientos de reembolso, deberían conducir a la fijación de precios más razonables y evitar la especulación, permitiendo que un mayor número de pacientes pudiera acceder a estos productos de primera necesidad que son los medicamentos. Pero lo cierto es que esto no es así, como alertan representantes de facultativos y pacientes, en especial, por lo que a las enfermedades raras se refiere.

propiedades considerablemente diferentes en cuanto a seguridad y/o eficacia, en cuyo caso el solicitante deberá facilitar datos suplementarios para demostrar la seguridad y/o eficacia de la diversidad de sales, ésteres o derivados presentes en una sustancia activa autorizada. Las diferentes formas farmacéuticas orales de liberación inmediata se considerarán una misma forma farmacéutica."

Destacados autores, y por todos ellos Valverde (2007, pp. 85-107), ponen en duda la eficacia de los medicamentos genéricos, criticando que, por un lado, se hable de sustancias activas frente a sustancias inertes; que, por otro, se afirme que, por norma, todos los derivados de una sustancia activa se considerarán una misma sustancia activa; o que, incluso, se acepte la posibilidad de que un medicamento biológico pueda ser similar a un producto biológico de referencia.

En todo caso, lo cierto es que en los últimos años las diferentes Administraciones sanitarias han puesto en ellos sus ojos con el fin de contener el crecimiento del gasto farmacéutico público. A este respecto, resulta muy significativo el tenor literal del art. 85.4 de la LGURMPS, en su redacción dada por el Real Decreto Ley 16/2012: "Cuando la prescripción se realice por principio activo (lo que se ha convertido en la norma general dentro de nuestro Sistema Nacional de Salud), el farmacéutico dispensará el medicamento de menor precio de su agrupación homogénea y, en el caso de igualdad, el medicamento genérico o el medicamento biosimilar correspondiente". La Comunidad Autónoma de Andalucía ha sido un referente en la implantación de este tipo de políticas. 
Este entramado normativo está repercutiendo gravemente en muchas familias que sufren enfermedades de alto impacto económico, llegando a verse excluidas del sistema sociosanitario $^{2}$ (Barranco Vela, 2013 y 2014, Cantero Martínez, 2014 y Garrido Cuenca, 2014). Es más, medidas de copago que pueden llegar a ser justificables en otros ámbitos, suponen un importante varapalo para muchos enfermos raros que sobreviven - o viven con un mínimo de calidad - gracias a estos productos. Lo que recientemente ha denunciado con rotunda claridad una Sentencia del Tribunal Superior de Justicia de Galicia ${ }^{3}$ (España, 2013).

El acceso al medicamento se ve condicionado por estos factores, y en especial, por la búsqueda de rentabilidad económica perseguida por la industria farmacéutica. En nuestra sociedad actual, ha quedado invertida por completo la secuencia propia de lo que se ha dado en llamar el paradigma Galileo, supeditándose "la investigación científica a los objetivos de explotación técnica y rentabilidad económica" (Esteve Pardo, 2009, p. 66 y ss.). Es lo que se conoce como tecnociencia. Las decisiones en materia de investigación científica (líneas de investigación, objetivos, métodos...) se toman hoy día - también en el campo del

\footnotetext{
${ }^{2}$ Piénsese, por ejemplo, en la Resolución de 10 de septiembre de la Dirección General de Cartera Básica de Servicios del Sistema Nacional de Salud y Farmacia, desarrollando lo previsto en el Real Decreto Ley 28/2012, equiparando las dispensaciones mediante receta médica y las órdenes de dispensación hospitalaria a efectos de la aportación en la prestación farmacéutica. El objeto de esta norma es someter a los criterios de la aportación reducida a los medicamentos de dispensación ambulatoria en el hospital, esto es, aquellos que sin tener la calificación de uso hospitalario tienen limitada su dispensación en el ámbito del Sistema Nacional de Salud en los servicios de farmacia de los hospitales. Estos medicamentos son fundamentalmente los indicados par las "enfermedades graves o crónicas" (cáncer, tumores cerebrales, leucemias, intolerancias graves a la lactosa, esclerosis múltiple o hepatitis C). Estos medicamentos (50) pasan de estar exentos de aportación a estar sometidos a un copago del $10 \%$, con un límite de aportación reducida de $4,20 €$. Esto puede suponer para un enfermo raro $400 / 500 €$ al mes, pues la mayoría de ellos multiplican esta cifra por la polimedicación a la que están sujetos. ¿Qué sentido tiene esta medida? ¿Carácter disuasorio? ¿Carácter recaudatorio? ¿Ahorro?...

${ }^{3}$ Me permito transcribir su Fundamento Jurídico 22: "No puede acaecer sin embargo nunca lo sucedido en el presente caso donde no sólo la Administración sanitaria prescindió del criterio médico - a la postre inclusive científicamente confirmado -, sino que se interpuso entre el facultativo correspondiente y su paciente, sin que autorizase la dispensación de aquel medicamento pese a estar oficialmente homologado y autorizado y haber sido en su día prescrito mediante razonables y razonados pareceres facultativos oficiales nunca de contrario desmentidos que le fueron sucesivamente expuestos y sin que su patente inactividad - en realidad su denegación de facto-, tuviese otra presumible motivación que el ahorro, incurriendo en cualquier caso así las Autoridades de la Consellería de Sanidad de la Xunta de Galicia y de aquel Ente institucional-autonómico antes mencionadas inclusive en desviación de poder $[\cdots]$, sin perjuicio de que en el presente caso conlleve también mediatamente un radical defecto anulatorio al afectar incluso al ejercicio de aquel derecho fundamental individual tutelado por el Art. 15 "ab initio" de la Constitución, [‥] en la medida en que -tal como antes ya pormenorizadamente se referenció-, jurisprudencial y constitucionalmente se ha privado a la Administración sanitaria de la facultad de denegar o restringir la asistencia sanitaria y medicamentosa - máxime si se trata de un medicamento único sin alternativa farmacológica alguna en el mercado -, por razón de dificultades presupuestarias o de ahorro económico".
} 
medicamento - atendiendo a unos muy estudiados cálculos coste-beneficio, que deciden, en último término, el que se investigue y produzca un determinado fármaco ${ }^{4}$.

Muy ilustradoras al respecto son las manifestaciones, hace treinta años, del entonces director ejecutivo de Merck, Henry Gadsten, afirmando sin tapujos a la revista Fortune que su sueño sería fabricar medicamentos para gente sana: «vender a todo el mundo», como lo hacía el fabricante de chicles Wringley's (Moynihan y Cassels, 2006, p. 9) ${ }^{5}$ y, hace escasos meses, de Marijn Dekkers, Consejero Delegado de Bayer: "No creamos este medicamentos (el anticanceroso Nexavar) para los indios, sino para los occidentales que puedan pagarlo" (De Benito, 2014).

Por último, por sólo reseñar algunas de las razones que bajo mi punto de vista más incidencia tienen en este ámbito, diez grupos farmacéuticos poseen el $50 \%$ del mercado mundial de medicamentos, los llamados Big Pharma, laboratorios con derechos exclusivos para fabricar y comercializar estos singulares productos. La globalización del sistema de patentes y la estructura monopolística del mercado farmacéutico han convertido al paciente en prisionero del mercado.

Los intereses económicos en juego han propiciado que el derecho a la propiedad intelectual pueda prevalecer sobre el derecho a la protección de la salud. Buena prueba de ello es la aplicación que se ha dado en los últimos años, en particular, en los países en vías de desarrollo (Bombillar Sáenz, 2013a), al Acuerdo sobre los Aspectos de los Derechos de Propiedad Intelectual relacionados con el Comercio (ADPIC) de la Organización Mundial del Comercio ${ }^{6}$. Por tanto, y aún

\footnotetext{
${ }^{4}$ Muy atrás en el tiempo quedó aquel período en el que el científico, con total libertad, se empeñaba en desentrañar los secretos de la naturaleza, en la búsqueda del conocimiento, y luego, accesoriamente, llegado el caso, en la rentabilización económica de su hallazgo. Ahora, por el contrario, desde el primer momento, la investigación va enfocada precisamente a ese objetivo: a la extracción de la máxima rentabilidad económica de los productos que surjan de la aplicación de esos conocimientos. Un planteamiento que responde sin ambages a una lógica empresarial, y que va más allá de la concepción clásica de la libertad de investigación científica.

${ }^{5}$ Es el caso de las conocidas como píldoras de la felicidad, en relación con aquellas enfermedades propias del estilo de vida de nuestra sociedad, como el estrés o la depresión. La industria farmacéutica ha conseguido redefinir y ampliar el concepto de enfermedad, transformando en enfermedad estados de la vida cotidiana no necesariamente patológicos (menopausia, timidez...), con el objetivo de expandir el mercado y sus ganancias, vendiendo unos medicamentos que no requieren grandes dosis de investigación o innovación tecnológica a pacientes que realmente no los necesitan; y que, precisamente por el consumo de los mismos, pueden arriesgar su salud, al verse afectados por los efectos secundarios de éstos (Moynihan y Cassels, 2006).

${ }^{6}$ El sistema de patentes que, en su origen, buscaba - como ponía de relieve la Ley de Patentes de la República de Venecia de 1474, la primera ley de patentes de la historia - obligar a los titulares de éstas a publicar sus invenciones, para que las mismas no fuesen secretas y todos se beneficiasen de ellas, ha desembocado en un sistema donde los laboratorios se sirven de todo tipo de artimañas para, en vez de desarrollar inventos genuinos, obtener patentes sobre adiciones secundarias al conocimiento que les permitan extender su monopolio sobre un producto o proceso concreto.
} 
reconociendo el importante papel que la industria farmacéutica juega en pro de la salud de la ciudadanía, y el innegable trabajo que realizan los científicos y demás personal que en ella se integra, no se puede negar el hecho de que los laboratorios farmacéuticos buscan, como cualquier otra empresa, la rentabilidad económica.

Las cifras hablan por si solas: el 14\% de la población mundial, el que vive en los países del primer mundo, consume el $80 \%$ de los medicamentos. La industria no investiga - al menos, no lo suficiente - para producir nuevos medicamentos que sirvan para atajar los problemas de salud pública de este $86 \%$ restante ${ }^{7}$. ONGs, como Farmamundi, alertan de la carencia de tratamientos eficaces y seguros para enfermedades tropicales que afectan y matan a millones de personas cada año (Farmamundi, 2002). La crisis del Ébola ha vuelto a poner, una vez más, todas estas carencias encima de la mesa.

Pero no hemos de salir fuera de las fronteras europeas para encontrarnos con problemas de acceso al medicamento. En Europa, millones de ciudadanos tienen aún hoy problemas para acceder a medicamentos esenciales para su salud, en especial, los cerca de treinta millones - 3 millones de ellos españoles -, que padecen una enfermedad rara (Barranco Vela, 2013 y 2014). No causa extrañeza el hecho de que la industria farmacéutica, que sabemos se desvive por conquistar nuevos clientes y mercados, sea poco propensa a elaborar medicamentos para el tratamiento de enfermedades poco frecuentes, pues éstas conllevan una escasa rentabilidad por el reducido número de afectados y el elevado gasto en investigación e innovación que generan (Alba Romero y Gutiérrez Pérez, 1998 o Panunzio y Recchia, 2007).

En este contexto, y bajo estas premisas, vamos a analizar aquí brevemente el régimen jurídico europeo que afecta a los medicamentos huérfanos, las políticas públicas emprendidas en España en los últimos años para, en este marco comunitario de referencia, atender a las necesidades de las personas afectadas por enfermedades raras, así como, por último, los muchos retos que aún quedan por

Un sistema perverso ante el que se rebeló Sudáfrica en el año 2000, razón por la que fue demandada por treinta y nueve empresas farmacéuticas. La presión pública suscitada por estos hechos llevó a la OMC a aprobar en Doha, en 2001, una Declaración en la que se aboga por una interpretación del ADPIC acorde con el derecho a la protección de la salud pública y, en particular, con la promoción del acceso universal a los medicamentos.

Brasil fue el primer país en acogerse a las cláusulas de Doha, en el marco de su programa de lucha contra la pandemia del SIDA.

${ }^{7}$ Un tercio de la población mundial, dos mil millones de personas, el que vive en los países en vías de desarrollo, se ve afectado en la actualidad por enfermedades (el botulismo, la malaria, la enfermedad del sueño, la enfermedad de Chagas, la leishmaniasis, la tuberculosis o la lepra) que son curables en el llamado primer mundo, sin poder acceder a un tratamiento eficaz por falta de recursos económicos. 
resolver para conseguir garantizar a este colectivo el derecho a la protección de la salud en paridad de condiciones con el resto de ciudadanos.

\section{Régimen jurídico de los medicamentos huérfanos}

\subsection{El concepto jurídico de medicamento huérfano y de enfermedad rara.}

Un medicamento huérfano es aquel medicamento destinado al diagnóstico, prevención o tratamiento de una enfermedad rara o poco frecuente (Barranco Vela, 2010a y 2010b, y Bombillar Sáenz, 2010a, pp. 279 y ss.). La definición de medicamento huérfano está ligada irremediablemente al concepto que normativamente se acuñe de lo que es una enfermedad rara. Se entiende por enfermedad rara - en el ámbito de la Unión Europea - aquella que pone en peligro la vida o conlleva una incapacidad crónica, presentando una prevalencia inferior a 5 personas por cada 10.000. Podrá superarse esta cifra - y seguir encuadrándose tal enfermedad dentro de esta calificación - cuando no sea posible para un laboratorio farmacéutico recuperar a través de sus ventas en el territorio nacional los costes de desarrollo y distribución ${ }^{8}$.

Las enfermedades raras han sido declaradas un sector prioritario de acción comunitaria en el ámbito de la salud pública (Garrido Cuenca, 2013). Son enfermedades crónicas de origen genético, que se suelen manifestar en la niñez y que afectan a la autonomía personal de quienes las padecen, produciendo una dolorosa carga psico-social para los afectados y sus familiares, origen de problemas educativos, laborales y sociales. Carga que se agrava ante la dificultad de encontrar tratamientos adecuados (que no realmente efectivos), ya que aún existe un gran desconocimiento y desinformación sobre ellas dada su complejidad médica y su baja rentabilidad económica ${ }^{9}$.

En la actualidad, se contabilizan entre unas 6.000 y 7.000 enfermedades raras con peligro de muerte o invalidez crónica; manejándose a nivel europeo la cifra de un enfermo por cada 2.000 habitantes para cada patología (es decir, un máximo de 227.000 pacientes en la Unión y 22.000 en España). No obstante, estas

\footnotetext{
8 Siempre que su promotor pueda demostrar que resultará improbable que, sin incentivos, la comercialización de dicho medicamento en la Unión Europea genere suficientes beneficios para justificar la inversión necesaria; del mismo modo, no debe existir ningún método satisfactorio autorizado en la Unión para una de estas patologías que va a tratar el medicamento o, existiendo, el nuevo medicamento debe aportar un beneficio significativo a quienes la padecen.

${ }^{9}$ Como la Osteogénesis Imperfecta (o enfermedad de los huesos de cristal), por ejemplo.
} 
enfermedades raras afectarían, en su conjunto, en toda la Unión Europea, a unos treinta millones de personas; y, en España, en torno a tres. En nombre de esos más de tres millones de afectados actúa la Federación Española de Enfermedades Raras (FEDER). Estos afectados tienen, como cualquier otro ciudadano, derecho a acceder a medicamentos cuya calidad, seguridad y eficacia sean equivalentes a las de los medicamentos de que se benefician los demás pacientes.

En este ámbito, la patente no puede jugar el mismo papel que desempeña en otros campos, no puede desplegar todo su abanico de posibilidades, ya que el problema de fondo es, precisamente, la falta de un mercado potencial (Ficicchia, 2007). De ahí el empleo del calificativo de medicamentos "huérfanos" ${ }^{10}$ por parte de Henry Waxman, promotor de la Orphan Drug Act estadounidense; equiparando la labor a llevar a cabo con este tipo de medicamentos con la que la sociedad ha de desempeñar con los niños que carecen de padre y madre.

\subsection{El procedimiento de designación como medicamento huérfano. El Comité de Medicamentos Huérfanos de la EMA.}

A los medicamentos huérfanos les afectan, en especial, las siguientes normas: el Reglamento (CE) n. 141/2000, del Parlamento Europeo y del Consejo, de 16 de diciembre de 1999, sobre medicamentos huérfanos (Unión Europea, 2000); el Reglamento (CE) n. 847/2000, de la Comisión, de 27 de abril de 2000, por el que se establecen las disposiciones de aplicación de los criterios de declaración de los medicamentos huérfanos y la definición de los conceptos de "medicamento similar" y "superioridad clínica"; el Reglamento (CE) n. 726/2004, del Parlamento Europeo y del Consejo, de 31 de marzo de 2004, por el que se establecen procedimientos comunitarios para la autorización y el control de los medicamentos de uso humano y veterinario y por el que se crea la Agencia Europea de Medicamentos (Unión Europea, 2004); el Reglamento (CE) n. 507/2006, de la Comisión, de 29 de marzo de 2006, sobre la autorización condicional de comercialización de los medicamentos de uso humano que entran en el ámbito de aplicación del Reglamento (CE) n. 726/2004 del Parlamento Europeo y del Consejo (Unión Europea, 2006); y la Directiva n. 2001/83/CE, del Parlamento Europeo y del Consejo, de 6 de noviembre de 2001, por la que se establece un código comunitario sobre medicamentos para uso humano.

\footnotetext{
10 "Falto de algo, y especialmente de amparo", según la tercera acepción del Diccionario de la Real Academia Española.
} 
El Reglamento (CE) n. 141/2000 crea un procedimiento comunitario "abierto y transparente" para designar los medicamentos huérfanos y establece incentivos para la investigación, el desarrollo y la comercialización de estos medicamentos, especialmente mediante la concesión de un derecho de exclusividad comercial de diez años duración. La Unión Europea adoptó así en el año 2000, casi veinte años después que Estados Unidos, su propio régimen de incentivos al desarrollo de medicamentos huérfanos.

En el seno de la Agencia Europea de Medicamentos (EMA) contamos con un Comité de Medicamentos Huérfanos (COMP, de acuerdo con sus siglas en inglés) ${ }^{11}$ que se encarga de examinar las solicitudes de declaración de medicamentos como "medicamentos huérfanos", de aconsejar a la Comisión sobre la elaboración y la aplicación de una política comunitaria sobre este tipo de medicamentos y de asistir a ésta en sus contactos internacionales y con los grupos de apoyo a los pacientes, además de en la elaboración de unas directrices detalladas sobre los mismos.

Recordemos, con carácter general, que, en el plano organizativo, el modelo de agencia se ha consolidado como la forma de organización más adecuada para gobernar el mercado farmacéutico y alimentario ${ }^{12}$. La Agencia Europea de Medicamentos (EMA) y la Autoridad Europea de Seguridad Alimentaria (EFSA) se conciben como organismos, integrados por expertos independientes, donde la razón científico-técnica y la búsqueda del interés general se imponen frente a espurios intereses políticos o económicos; en el marco de un sistema administrativo integral, en red, que acoge a las diferentes agencias nacionales de los Estados miembros (Bombillar Sáenz, 2013c o Salvatore, 2012).

La designación de un determinado medicamento como "huérfano" conllevará que éste pueda acogerse al régimen especial contemplado al respecto en la

\footnotetext{
${ }^{11}$ Este Comité está compuesto por un miembro nombrado por cada uno de los Estados miembros, tres miembros nombrados por la Comisión para representar a las asociaciones de pacientes y tres miembros nombrados por la Comisión previa recomendación de la Agencia.

${ }^{12}$ La EMA y la EFSA auxilian a la Comisión en la realización de tareas reguladoras, pero sin tener conferidas las mismas, mediante informes de inspección o a través de dictámenes y recomendaciones que constituyen la base técnica y científica de las decisiones de la Comisión; dando lugar a una separación funcional y estructural de la organización encargada de la determinación o evaluación del riesgo con respecto a aquella otra a la que se le asigna su gestión. Sendas agencias facilitan el funcionamiento del mercado interior, aligeran el trabajo de la Comisión y promocionan - a través del trabajo en red que coordinan - una mayor uniformidad entre las políticas nacionales y europeas en el sector farmacéutico y alimentario. Por ello, siguiendo a Vírgala, ambas dos se pueden calificar como agencias "cuasi-reguladoras" o "pre-decisorias", ya que, como hemos apuntado, sus recomendaciones científicas tienen una influencia decisiva, real, de cara a la adopción de la decisión final por la Comisión, quien ratifica sistemáticamente sus recomendaciones (Vírgala Foruria, 2011).
} 
normativa comunitaria. Régimen en el que se dan cita lo que la doctrina ha dado en calificar push programmes y pull programmes: medidas tendentes, por un lado, a incentivar la inversión en investigación y desarrollo de la industria farmacéutica en este campo y, por otro, a propiciar la rápida puesta en el mercado de los correspondientes medicamentos huérfanos.

Para obtener la declaración de "medicamento huérfano", el promotor presentará una solicitud a la Agencia en cualquier fase del desarrollo de su medicamento, aunque siempre antes de la solicitud de autorización previa a la comercialización ${ }^{13}$, demostrando que cumple con los requisitos establecidos en la normativa comunitaria. El procedimiento consta de tres fases fundamentales: el control preventivo de la EMA, quien comprobará la validez de la solicitud y preparará un informe sucinto, la evaluación del COMP y la decisión final de la Comisión. El medicamento declarado "huérfano" se inscribirá en el correspondiente Registro.

Este procedimiento no está valorando la seguridad o calidad del medicamento en cuestión, si no si el mismo se ajusta a los parámetros que han de cumplir los medicamentos huérfanos, siendo uno de los más cuestionados el relativo a su eficacia con respecto a otros tratamientos ya existentes. En este marco de actuación, donde la Administración dispone, por lógica, de una amplia capacidad de apreciación, como apunta el propio TJUE en el asunto Now Pharma c. Comisión, cabe exigir, más si cabe, un plus de respeto de las garantías reconocidas por el ordenamiento jurídico comunitario en los procedimientos administrativos, exigiendo la cualificación e imparcialidad de todos los expertos involucrados (Bombillar Sáenz, 2011).

La Comisión hizo balance de la aplicación del Reglamento (CE) n. 141/2000, cinco años después de que éste viera la luz. Un balance muy positivo. Durante ese período (abril 2000-abril 2005) se presentaron 458 solicitudes de designación de medicamentos huérfanos ante la Agencia, resultando designados 268 productos. Del total de productos designados, veintidós obtuvieron autorización de comercialización, abarcando veinte diferentes patologías, para ocho de las cuales (40\%) no había, con anterioridad a dicha autorización, tratamiento alguno; mientras que para las doce restantes, los productos autorizados supusieron claros beneficios significativos a los

\footnotetext{
${ }^{13}$ El laboratorio farmacéutico podrá solicitar también la designación como medicamento huérfano para un medicamento que se encuentre ya en comercialización, pero que hubiese sido autorizado para una indicación terapéutica diferente; lo que suele ocurrir normalmente.
} 
pacientes. Se estima, por todo ello, que más de un millón de pacientes se han visto beneficiados por las medidas de este Reglamento ${ }^{14}$.

\section{a. Procedimiento centralizado de autorización de comercialización y posibilidad de autorización condicional.}

La autorización de comercialización de los medicamentos huérfanos se llevará a cabo siguiendo el conocido como "procedimiento centralizado" (Bombillar Sáenz, 2010 a, p. 342 y ss.), obligatorio para estos medicamentos desde 2005 de acuerdo con el referido Reglamento (CE) n. 726/2004, siendo la EMA la encargada de coordinar la evaluación científica de la calidad, seguridad y eficacia de estos medicamentos en el marco de este procedimiento comunitario de autorización.

El procedimiento centralizado permite una importante "economía procedimental", evitando el peregrinar de la industria farmacéutica Estado por Estado para conseguir la autorización del producto farmacéutico. El medicamento se somete a un solo proceso de evaluación, con el consiguiente beneficio para la industria y la sociedad. Esto no sólo ayuda a la creación de un mercado interior de medicamentos, sino que redunda en la protección de la salud pública.

De este modo, las solicitudes de comercialización por parte de los promotores de medicamentos huérfanos se presentarán directamente a la EMA, y no a las Administraciones sanitarias nacionales. El solicitante obtendrá una autorización que le reportará los mismos derechos y obligaciones en cada uno de los Estados miembros de la Unión Europea. El medicamento podrá ser desde entonces comercializado en cualquier punto de la Unión. No obstante, lamentablemente, no por el hecho de contar con dicha autorización centralizada, éste estará disponible al mismo tiempo en las oficinas de farmacia de todos los Estados miembros; una consecuencia de que las competencias en materia de prestación farmacéutica no se hayan comunitarizado.

Los medicamentos huérfanos pueden también acogerse a lo previsto en la norma europea en donde se aborda la autorización condicional de medicamentos (Unión Europea, 2006), que permite que, para satisfacer necesidades no cubiertas de los pacientes y en interés de la salud pública, se concedan autorizaciones de

\footnotetext{
14 En enero de 2005, fueron 198 los medicamentos designados y 16 los autorizados; asistiendo a un aumento del 25/30\% por año. En mayo 2006, las cifras serían 557 solicitudes presentadas, 360 medicamentos designados como medicamentos huérfanos y 25 autorizados para ser comercializados.
} 
comercialización basadas en datos clínicos "menos completos de lo habitual”, en los que no esté plenamente documentada la inocuidad y eficacia del medicamento.

Dado que estos medicamentos están dando respuesta a necesidades médicas no cubiertas por el mercado o constituyen un avance terapéutico sustancial respecto a los tratamientos ya existentes, y con el fin de fomentar y posibilitar la pronta llegada de estos medicamentos al mercado (a los pacientes), se acorta el procedimiento de autorización en uno o dos años (en lugar de los diez años que normalmente éstos requerirían para ser comercializados si debieran cumplir con todos los requisitos que el ordenamiento jurídico exige).

No obstante, y debido a los riesgos que ello supone, se ven sometidas estas autorizaciones a unas obligaciones de farmacovigilancia más intensas de lo habitual (Doménech Pascual, 2009); a unas obligaciones específicas y a un seguimiento extraordinario; exigiéndose a sus titulares que completen o comiencen determinados estudios para confirmar que la relación beneficio-riesgo es favorable y despejar así cualquier duda sobre la calidad, seguridad y eficacia del medicamento ${ }^{15}$.

Estas autorizaciones tendrán un período de validez de un año, con posibilidad de renovación, a solicitar seis meses antes de la fecha de expiración de la autorización. Para garantizar que los medicamentos no se retiren del mercado mientras tanto, la autorización condicional extenderá su validez hasta el mismo día que se pronuncie la Comisión sobre su renovación (Moreno-Tapia y Sabater, 2006:21). En cualquier caso, lo cierto es que estamos ante una autorización provisional, con un período de validez de un año; y condicional (aunque no se pretende que lo siga siendo indefinidamente), a renovar o no anualmente en función del cumplimiento de los requisitos señalados.

Las autorizaciones condicionales se unen a otros procedimientos especiales de autorización ya presentes en el ordenamiento comunitario como la "evaluación acelerada", que reduce el tiempo de estudio de 210 a 150 días (Unión Europea, 2004, art.14.9), y que puede acumularse al propio procedimiento que estamos comentando; la autorización concedida "en circunstancias excepcionales" (Unión Europea, 2004,

\footnotetext{
${ }^{15}$ Los titulares de estas autorizaciones garantizarán una información adecuada a los pacientes y especialistas sobre el medicamento en cuestión, mencionando en el resumen de las características del producto y en el prospecto el carácter de su autorización; llevarán un registro detallado de las sospechas de reacciones adversas de las que tengan conocimiento, con el fin de garantizar la salud pública y la de los concretos pacientes afectados o emitirán informes periódicos en materia de seguridad a las autoridades competentes.
} 
art.14.8), éstas siempre condicionales, para aquellos casos en los que no concurran algunos de los requisitos previstos por el Derecho comunitario para otorgar una autorización condicional; y el uso compasivo que referiremos más abajo.

b. Otras medidas de estímulo de cara a la concesión o mantenimiento de una autorización de comercialización para un medicamento huérfano: el derecho de exclusividad comercial por diez años.

En cualquier caso, y con el fin de facilitar la concesión o el mantenimiento de dicha autorización, se establece la posibilidad de conceder una exención, al menos parcial, de las tasas debidas por el promotor del medicamento huérfano a la EMA por sus servicios. Por otro lado, antes de presentar una solicitud de autorización previa a la comercialización, el promotor de un medicamento huérfano podrá solicitar el dictamen de la EMA sobre los diversos ensayos ${ }^{16}$ y pruebas que deban realizarse para demostrar la calidad, la seguridad y la eficacia del medicamento; lo que se conoce como asistencia en la elaboración de protocolos.

Llegamos, por fin, a la que es, sin duda, la medida estrella del Reglamento (CE) 141/2000. Cuando se conceda una autorización previa a la comercialización para un medicamento huérfano, dicho medicamento se beneficiará de un derecho de exclusividad comercial de diez años. La Unión Europea se abstendrá de aceptar cualquier otra solicitud previa a la comercialización, de conceder una autorización previa a la comercialización o de atender una nueva solicitud de extensión de una autorización previa a la comercialización existente con respecto a un medicamento similar para la misma indicación terapéutica.

Aunque podría pensarse que una patente ya otorgaría a un medicamento una protección comercial adecuada, sin que fuese necesaria, por tanto, esta exclusividad comercial; puede suceder que el medicamento en cuestión no pueda ser objeto de protección a través de patente porque no sea nuevo u original o se trate de una sustancia natural difícil de patentar; o, sencillamente, que su patente hubiera ya finalizado, descubriéndose posteriormente la utilidad del medicamento para tratar indicaciones "huérfanas". En estos casos, la exclusividad comercial se convierte en algo muy atractivo para el laboratorio farmacéutico (Ficicchia, 2007, p. 449).

\footnotetext{
${ }^{16}$ Hemos de destacar que, en materia de ensayos clínicos, acaba de aprobarse el Reglamento (UE) $n$. 536/2014 del Parlamento Europeo y del Consejo de 16 de abril de 2014 sobre los ensayos clínicos de medicamentos de uso humano, y por el que se deroga la Directiva 2001/20/CE.
} 
No obstante, dicho período de exclusividad comercial podrá reducirse a seis años, si al finalizar el quinto se demuestra que el medicamento de que se trate ha dejado de cumplir los criterios recogidos en el Reglamento (CE) n. 141/2000 (por ejemplo, si se demuestra que la rentabilidad del producto es ya suficiente para no justificar el mantenimiento de la exclusividad comercial). Por otra parte, la citada exclusividad no impedirá la comercialización de un segundo medicamento cuando el titular de la autorización de comercialización del medicamento huérfano inicial: dé su consentimiento al segundo solicitante o no pueda suministrar suficiente cantidad de dicho producto; o también cuando el promotor del segundo medicamento pueda demostrar que éste es más seguro, eficaz o clínicamente superior.

\section{Repaso a algunas de las política públicas españolas a favor de los afectados por enfermedades raras.}

Los medicamentos huérfanos podrán beneficiarse de otras medidas de estímulo establecidas por la Comisión y los Estados miembros para fomentar la investigación, el desarrollo y la comercialización de estos medicamentos; en particular, de medidas de apoyo a la investigación en favor de pequeñas y medianas empresas. Estas medidas deberán comunicarse a la Comisión para su evaluación. De esta forma, el país que tenga más y mejores iniciativas en este campo se constituirá en ejemplo y modelo a seguir por el resto.

Desde la aprobación en el Senado, en febrero de 2007, del Informe de la Ponencia de estudio encargada de analizar la especial situación de los pacientes con enfermedades raras, aunque aún quede mucho camino por recorrer, España ha comenzado a implicarse de forma decidida en esta cuestión (Barranco Vela, 2013 y 2014). Prueba de ello fue la aprobación, en junio de 2009, por el pleno del Consejo Interterritorial del Sistema Nacional de Salud, de la Estrategia en enfermedades raras del Sistema Nacional de Salud. Esta Estrategia consta de siete líneas estratégicas, a saber: información sobre enfermedades raras, prevención y detección precoz, atención sanitaria, terapias, atención sociosanitaria, investigación y formación.

En el ámbito organizativo, hemos de destacar la creación por Orden SCO/3158/2003, de 7 de noviembre, incardinado en el Instituto de Salud Carlos III de 
Madrid, del Instituto de Investigación de Enfermedades Raras (IIER) ${ }^{17}$, un centro que sirve de referencia a la estructura sanitaria del Estado, donde, en colaboración con las Comunidades Autónomas, se realizan actividades relacionadas con este tipo de enfermedades (en el terreno de la investigación, el técnico-asistencial, la docencia o la información).

Además, con el fin de consolidar estructuras estables de investigación cooperativa de excelencia, tanto básica como clínica, de las que puedan beneficiarse los pacientes con enfermedades raras, se ha creado, también en el mencionado Instituto de Salud Carlos III de Madrid, el Centro de Investigación Biomédica en Red de Enfermedades Raras (CIBERER), uno de los nueve consorcios dependiente de este Instituto.

De todas las medidas puestas en práctica, la más esperada fue, sin duda, la construcción en Burgos del Centro de Referencia Estatal de Atención a Personas con Enfermedades Raras y sus Familias (Creer). Encuadrado dentro de la línea estratégica "Atención sociosanitaria", este centro se ha erigido como un centro de referencia en la promoción, desarrollo y difusión de conocimientos, experiencias innovadoras y métodos de atención a personas con enfermedades raras.

No sólo eso, este centro también se ocupa de prestar servicios de apoyo a familias y cuidadores; así como de promover la autonomía personal y la participación social de las personas que padecen este tipo de enfermedades, en conexión con lo previsto en Ley 39/2006, de 14 de diciembre, de Promoción de la Autonomía Personal y Atención a las personas en situación de dependencia. No en vano, la inmensa mayoría de los pacientes afectados por enfermedades raras son personas dependientes. Precisamente, el CRE de Burgos forma parte de la red de Centros de Referencia Estatal del IMSERSO que se enmarcan en esta Ley.

La vigente Ley del Medicamento (España, 2006) equipara, en su artículo 2, a los medicamentos huérfanos con los "medicamentos sin interés comercial" de su artículo 109.1, esto es: "aquéllos de los que existe ausencia o insuficiencia de suministro en el mercado nacional, siendo necesarios para el tratamiento de determinadas enfermedades o patologías". Es más, la Disposición Adicional Primera de esta norma señala que el Ministerio de Sanidad, con objeto de desarrollar e impulsar el suministro de medicamentos necesarios para la asistencia sanitaria, entre

\footnotetext{
${ }^{17}$ Este Instituto se funda sobre la base del preexistente Centro de Investigación sobre el Síndrome del Aceite Tóxico y Enfermedades Raras.
} 
otras cuestiones, promoverá la fabricación y comercialización de los medicamentos sin interés comercial, entre los que se encuadran los huérfanos. En este mismo sentido, en el ámbito autonómico, se pronuncia la Ley 22/2007, de 18 de diciembre, de Farmacia de Andalucía. Es por esta razón, y de cara a asegurar su abastecimiento, que el Gobierno podrá adoptar medidas especiales en relación con la fabricación, importación, distribución y dispensación de estos medicamentos; y emprender medidas relativas a su régimen económico y fiscal.

Entre las medidas acogidas por el legislador estatal para propiciar la consecución de esos objetivos destaca la exención de la tasa correspondiente a las prestaciones de servicios o a la realización de actividades relativas a la fabricación de este tipo de medicamentos; en conexión con la posibilidad, contemplada en el ámbito comunitario, de conceder una exención, al menos parcial, de las tasas debidas por el promotor de un medicamento huérfano a la EMA por sus servicios.

Mención aparte merece la incidencia en este campo del Real Decreto por el que se regula la disponibilidad del medicamento en situaciones especiales, esto es: el uso compasivo, de medicamento extranjero y de medicamento en condiciones distintas a las autorizadas (España, 2009), en desarrollo de lo dispuesto en el artículo 24 de la Ley del Medicamento, al que en adelante denominaremos RDDMSE.

El RDDMSE pretende facilitar el acceso a medicamentos en fase de investigación clínica a aquellos pacientes que no dispongan de una alternativa terapéutica satisfactoria que sufran una enfermedad gravemente debilitante o que ponga en peligro sus vidas, sin formar parte de un ensayo clínico (Núñez Lozano, 2006 y 2011). Esta norma también se ocupa del acceso a medicamentos autorizados en otros países pero no en España, que no estén en investigación y cuyo uso sea imprescindible para el paciente (los conocidos como "medicamentos extranjeros"); y de los medicamentos utilizados en condiciones distintas a las previstas en la ficha técnica, más conocido como uso off label (Blanco Reina, 2012).

El acceso a este tipo de medicamentos en situaciones especiales llevó en España en 2008, según informa nuestro Ministerio de Sanidad, a la tramitación de 60.000 solicitudes ante la AEMPS ${ }^{18}$. La mayoría de estas solicitudes recayeron sobre medicamentos oncológicos, neurológicos, oftalmológicos y dermatológicos. Es en el

\footnotetext{
18 En un 50\% referidas a medicamentos extranjeros, en un 35\% a medicamentos en condiciones distintas a las previstas en la ficha técnica y en un 15\% a medicamentos en investigación.
} 
campo de las enfermedades raras donde hay un mayor número de petición de autorizaciones de uso compasivo.

En relación con el uso compasivo ${ }^{19}$ (Bombillar Sáenz, 2010b y 2010c), el RDDMSE no contempla ya la necesidad de una solicitud y una autorización individualizada para cada paciente. El acceso a los medicamentos en investigación o no autorizados en España podrá gestionarse telemáticamente ${ }^{20}$ para cada paciente, y también a través de una autorización temporal emitida por la AEMPS, en coordinación con el resto de agencias europeas, para un grupo de pacientes. No es ya necesaria la autorización de la AEMPS para cada caso concreto. El recurso a estas autorizaciones temporales de utilización permite agilizar los trámites administrativos - algo a valorar especialmente en un ámbito donde se encuentran en juego situaciones clínicas bastante comprometidas. Los pacientes que reúnan los requisitos indicados en el protocolo podrán acceder al uso compasivo sin necesidad de recurrir a una autorización individualizada.

Este RDDMSE también aborda el problema del acceso a medicamentos utilizados en condiciones distintas a las autorizadas en España y recogidas en la ficha técnica $^{21}$. EI RDDMSE establece un procedimiento diferenciado de autorización para este tipo de supuestos, distinto del recogido para el uso compasivo. Esta situación especial se da, por ejemplo, en medicamentos "clásicos", que pueden presentar condiciones de uso establecidas en la práctica clínica, pero no contempladas en su autorización, al no resultar rentable económicamente llevar a cabo los estudios necesarios para obtener dicha autorización. Los médicos se encargarán de informar a los pacientes de los riesgos que dicho medicamento pueda suponer para su salud. La nueva normativa elimina la necesidad de obtener una autorización individual de la AEMPS, con independencia de su capacidad para emitir cuantas recomendaciones al respecto estime conveniente; recomendaciones que deberán ser tenidas en cuenta

\footnotetext{
${ }^{19}$ A efectos del RDDMSE, se entiende por uso compasivo de medicamentos en investigación: "utilización de un medicamento antes de su autorización en España en pacientes que padecen una enfermedad crónica o gravemente debilitante o que se considera pone en peligro su vida y que no pueden ser tratados satisfactoriamente con un medicamento autorizado. El medicamento de que se trate deberá estar sujeto a una solicitud de autorización de comercialización, o bien deberán estar siendo sometido a ensayos clínicos" (art. 2.1 RDDMSE).

${ }^{20}$ EI RDDMSE apuesta porque todas las solicitudes que tengan lugar a su amparo se presenten a la AEMPS, salvo en casos excepcionales, de forma telemática, conforme a lo establecido en el art. 27.6 de la Ley 11/2007, de 22 de junio, de acceso electrónico de los ciudadanos a los Servicios Públicos.

${ }^{21}$ EI RDDMSE define como uso de medicamentos en condiciones diferentes de las autorizadas: "el uso de medicamentos en condiciones distintas de las incluidas en la ficha técnica autorizada" (art. 2.2 RDDMSE).
} 
por los centros sanitarios a la hora de elaborar los pertinentes protocolos terapéuticos-asistenciales en sustitución de las autorizaciones individuales.

En cuanto a los medicamentos extranjeros ${ }^{22}$, la norma permite que los pacientes accedan a los mismos a través de una solicitud individualizada conforme a un protocolo avalado por la AEMPS; siempre y cuando estos medicamentos estén legalmente autorizados en otros países y resulten imprescindibles para la prevención, el diagnóstico o el tratamiento de patologías concretas, no existiendo en nuestro país alternativa adecuada autorizada para esa indicación; y no dándose los presupuestos para calificar dicha situación como de uso compasivo.

También tenemos que tener en cuenta en este terreno el dictado del Real Decreto 618/2007, de 11 de mayo, por el que se regula el procedimiento para el establecimiento, mediante visado, de reservas singulares a las condiciones de prescripción y dispensación de los medicamentos; así como las últimas medidas de ámbito estatal abogando por la contención del gasto público farmacéutico (por ejemplo, la Disposición final cuarta del Real Decreto-ley 4/2010, de 26 de marzo, de racionalización del gasto farmacéutico con cargo al Sistema Nacional de Salud, regula la dispensación de determinados medicamentos desde el ámbito hospitalario, potenciando su uso adecuado, su mayor vigilancia, supervisión y control).

A todo ellos de sumar el hecho de que algunas Comunidades Autónomas, como Valencia ${ }^{23}$ o Andalucía ${ }^{24}$, han dictado su propia normativa en desarrollo del RDDMSE.

\footnotetext{
${ }^{22}$ De acuerdo con el RDDMSE se entiende por acceso a medicamentos no autorizados en España: "la utilización de medicamentos autorizados en otros países pero no autorizados en España, cuando no cumplan con la definición de uso compasivo de medicamentos en investigación" (art. 2.2 RDDMSE).

${ }_{23}$ Véase la Orden 15/2010, de 17 noviembre (DOCV, n. 6417, de 15 de diciembre de 2010), en desarrollo de esta norma reglamentaria estatal, o el Decreto 118/2010, de 27 de agosto, del Consell (DOCV, n. 6347, de 3 de septiembre de 2010), que ordena y prioriza actividades de las estructuras de soporte para un uso racional de los productos farmacéuticos en la Agencia Valenciana de Salud. Allí juega un importante papel en este campo la Comisión Asesora de Utilización de Medicamentos en Situaciones Especiales (CAUME), junto con la Comisión de Farmacia y Terapéutica Hospitalaria y Comisión de Uso Racional del Medicamento.

${ }^{24}$ En Andalucía, por Resolución 369/09, de 7 de agosto de 2009, de la Dirección Gerencia del Servicio Andaluz de Salud (SAS) se crea la Comisión Asesora para la armonización de los criterios de utilización de medicamentos en condiciones diferentes a las establecidas en su ficha técnica. Se establece aquí un procedimiento unificado de autorización previa para la preinscripción y dispensación por las oficinas de farmacia de los medicamentos cuyas recetas estén sometidas al control de visado cuando, excepcionalmente, por carecer de alternativas terapéuticas para un determinado paciente, se requiera su utilización en condiciones diferentes a las establecidas en su ficha técnica. El facultativo que proceda de tal modo habrá de respetar las condiciones generales recogidas en el RDDMSE y las específicas de la autorización correspondiente.
} 
No mucho más se puede decir hasta que no veamos los resultados que en la práctica este RDDMSE produzca. En cualquier caso, es una norma esperanzadora para los millones de pacientes que llevan luchando titánicamente durante años contra su propia enfermedad y contra un procedimiento lento, farragoso y burocratizado hasta el extremo. Una carrera de obstáculos contrarreloj para acceder a un medicamento que les permita salvar o mejorar considerablemente su bien más preciado: la vida.

\section{Problemas aún por resolver en materia de acceso al medicamento huérfano y perspectivas de futuro.}

Aún queda mucho camino por recorrer para facilitar el acceso de los pacientes a los medicamentos huérfanos: los excesivamente largos períodos de espera, las diferencias existentes entre los diversos sistemas de reembolso, la escasa experiencia prescriptiva de los médicos en este ámbito, la diferencia de precios según el país en cuestión (del 25\% al 70\%); o que éstos sólo se puedan adquirir, con carácter general, en el ámbito hospitalario. Son sólo algunas de las cuestiones que se pueden reseñar. Aunque, sin duda, el principal problema en esta materia continúa siendo el de la prestación farmacéutica.

La designación de un medicamento como medicamento huérfano, el protocolo de asistencia a los promotores de los mismos y la autorización de comercialización son procesos centralizados en donde la EMA y la Comisión llevan las riendas; pero la evaluación de valor terapéutico, el precio y el reembolso de estos medicamentos (la prestación farmacéutica) sigue siendo responsabilidad de cada Estado miembro, no es una competencia que haya sido comunitarizada. Este reparto de competencias impide la creación de un mercado único de medicamentos huérfanos y, por ende, juega en contra de las personas afectadas por enfermedades raras.

La doctrina sigue buscando nuevas vías de actuación en este campo. En este sentido, se ha propuesto consentir a los laboratorios farmacéuticos, bajo la tutela del correspondiente organismo público, el traspaso de los derechos de exclusividad comercial a otro producto de su elección; la institución con dinero público - en conexión con el modus operandi existente en materia de enfermedades "olvidadas" de fondos para la adquisición de medicamentos huérfanos; o la posibilidad de atribuir 
a este tipo de fármacos un derecho de examen prioritario, pudiendo el laboratorio titular de los mismos transferir dicho derecho de prioridad a otro de sus medicamentos. De entre todas estas iniciativas, aunque sin respaldo legal expreso en la normativa comunitaria, resulta especialmente novedosa e interesante la apuntada por Ficicchia: hacer posible que un medicamento designado como huérfano en Estados Unidos se reconozca automáticamente como tal en la Unión Europea, creando un status internacional para este tipo de medicamentos, que uniformice todas las particularidades nacionales hasta ahora existentes (Ficicchia, 2007:453). De hecho, en esta línea trabajan ya las tres grandes agencias reguladoras del medicamento a nivel mundial: la EMA, la FDA estadounidense y la PMDA japonesa.

\section{Referencias}

ALBA ROMERO, Susana y GUTIÉRREZ PÉREZ, María Victoria. Aspectos jurídicossanitarios de las enfermedades poco frecuentes y los medicamentos huérfanos en la Unión Europea. Revista de Derecho Comunitario Europeo, 2(4):667-682, Jul-Dic 1998. [on line] ISSN: 1138-4026. Disponible en http://www.cepc.gob.es/publicaciones/revistas/revistaselectronicas?IDR=4\&IDN=287\& IDA=9641 [Fecha de consulta: 1 sep. 2014]

BAES, Celine. El uso racional del medicamento. Fundamento de la intervención administrativa en el sector del medicamento. Tesis (doctorado). Granada, Universidad de Granada, Facultad de Derecho, 2010. [on line] Disponible en http://digibug.ugr.es/handle/10481/19733\#.U vQZKMjWII [Fecha de consulta: 1 sep. 2014]

BARRANCO VELA, Rafael. Una nuova sfida per il diritto alla tutela della salute: l'accesso dei pazienti con malattie rare ai medicinali in Spagna. En SESTA, Michele. (Dir.). L'erogazione della prestazione medica tra diritto alla salute, principio di autodeterminazione e gestione ottimale delle risorse sanitarie. Rimini: Maggioli, 2014. 806 p. ISBN: 9788891603609.

Un nuevo desafío para el derecho a la protección de la salud: el acceso al medicamento en España de los pacientes con enfermedades raras. En: PÉREZ MIRAS, Antonio; TERUEL LOZANO, Germán y RAFFIOTTA, Edoardo (Dirs.). Desafíos para los derechos de la persona ante el siglo XXI: Vida y ciencia. Cizur Menor (Navarra): Thomson-Aranzadi, 2013, 448 p. ISBN: 978-84-9014-555-5.

El estatuto jurídico de los medicamentos huérfanos en la Unión Europea: El derecho a la salud de los pacientes con enfermedades raras. En: BARRANCO VELA, Rafael (Dir.) y BOMBILLAR SÁENZ, Francisco Miguel (Coord.). El acceso al medicamento: retos jurídicos actuales, intervención pública y su vinculación al derecho a la salud. Granada: Comares, 2010. 240 p. ISBN: 978-849836-636-5.

Legal regulation on orphan drugs ten years after the Communitarian regulations were passed, Pharmaceuticals Policy and Law, Amsterdam, 12(3-4): 217-228, Jan 2010. ISSN: 1389-2827. 
BARRANCO VELA, Rafael (Dir.) y BOMBILLAR SÁENZ, Francisco Miguel (Coord.). El acceso al medicamento: retos jurídicos actuales, intervención pública y su vinculación al derecho a la salud. Granada: Comares, 2010. 240 p. ISBN: 978-849836-636-5.

BERGEL, Salvador Darío. Bioética y el derecho humano de acceso a los medicamentos. En: PALACIOS, Marcelo (Ed.). Actas del IV Congreso Mundial de Bioética. Gijón: SIBI, 2005. 728 p.

BLANCO REINA, Encarnación. Disponibilidad de medicamentos en situaciones especiales: uso compasivo y uso off-label. Actualidad en Farmacología y Terapéutica, Madrid, 10(1):43-49, Mar 2012. ISSN: 16984277.

BOMBILLAR SÁENZ, Francisco Miguel. Patentes farmacéuticas y derecho de acceso al medicamento: un viaje desde la República de Venecia a la de Sudáfrica, pasando por la ciudad de Doha. Direito Público, Brasilia, (54):31-44, Nov-Dez 2013. ISSN: 1806-8200.

Intervención administrativa de orden público sanitario y régimen jurídico del medicamento. En: LARIOS RISCO, David, GONZÁLEZ GARCÍA, Lola y DE MONTALVO JÄÄSKELÄINEN, Federico (Coords.); PALOMAR OLMEDA, Alberto y CANTERO MARTÍNEZ, Josefa (Dirs.). Tratado de derecho sanitario, Cizur Menor (Navarra): Thomson-Aranzadi, 2013, 2 vols., 1146 p, ISBN: 9788490146569.

Estructura, organización y funciones de la Administración de la Unión Europea en materia de salud pública. Dos ejemplos: la Agencia Europea de Medicamentos y la Autoridad Europea de Seguridad Alimentaria. En: PÉREZ GÁLVEZ, Juan Francisco y BARRANCO VELA, Rafael (Dirs.). Derecho y salud en la Unión Europea. Granada: Comares, 2013. 376 p. ISBN: 9788490450710.

El derecho a una buena administración. Acerca de la supuesta falta de cualificación e imparcialidad de los miembros que componen los comités de expertos que auxilian a la Administración en el desempeño de su labor: Comentario a la sentencia del Tribunal de Primera Instancia de las Comunidades Europeas (sala quinta), de 9 septiembre 2010, asunto Now Pharm AG contra Comisión Europea. En: PÉREZ ZAFRILLA, Pedro, SARRIÓN ESTEVE, Joaquín y BENLLOCH DOMÉNECH, Cristina (Coords.). Construyendo ciudadanía: teoría y praxis. Granada: Comares, 2011. 370 p. ISBN: 9788498368505.

Intervención administrativa y régimen jurídico del medicamento en la Unión Europea. Tesis (doctorado). Granada, Universidad de Granada, Facultad de Derecho, 2010.2 [on line] Disponible en http://digibug.ugr.es/handle/10481/4863\#.U vQsKMjWII [Fecha de consulta: 1 sep. 2014]

The 'Compassionate Exemption' in Spain: Not Asking for Compassion. Opinio Juris in Comparatione, Pisa, 2(1):1-25, Sept 2010. [on line] Disponible en: http://ssrn.com/abstract=1647593 [Fecha de consulta: 1 sep. 2014].

El acceso a medicamento en situaciones especiales. Uso compasivo, uso en condiciones distintas a las autorizadas y medicamento extranjero. En: BARRANCO VELA, Rafael (Dir.) y BOMBILLAR SÁENZ, Francisco Miguel (Coord.). El acceso al medicamento: retos jurídicos actuales, intervención pública y su vinculación al derecho a la salud. Granada: Comares, 2010. 240 p. ISBN: 978-849836-636-5. 
CANTERO MARTÍNEZ, Josefa. Constitución y derecho a la protección de la salud. En: BALAGUER CALLEJÓN, Francisco y ARANA GARCÍA, Estanislao. Libro homenaje al profesor Rafael Barranco Vela. Cizur Menor (Navarra): Thomson Reuters-Civitas, 2014. 3208 p. ISBN: 9788447049080.

CIBERER. Centro de Investigación Biomédica en Red de Enfermedades Raras. [on line] Disponible en http://www.ciberer.es/ [Fecha de consulta: 1 sep. 2014]

CREER. Centro de Referencia Estatal de Atención a Personas con Enfermedades Raras y sus Familias de Burgos. [on line] Disponible en http://www.imserso.es/creer 01 [Fecha de consulta: 1 sep. 2014]

DE BENITO, Emilio. No creamos medicamentos para indios, sino para los que pueden pagarlo. El País, Madrid, 24 en. 2014 (reportaje de). Disponible en: http://sociedad.elpais.com/sociedad/2014/01/23/actualidad/1390497913 508926.html

[Fecha de consulta: 1 sep. 2014]

DOMÉNECH PASCUAL, Gabriel. El régimen jurídico de la farmacovigilancia. Cizur Menor (Navarra): Thomson-Aranzadi, 2009, 379 p. ISBN: 9788447031559.

EMA. European Medicines Agency. [on line] Disponible en www.ema.europa.eu [Fecha de consulta: 1 sep. 2014]

ESPAÑA. Tribunal Superior de Justicia de Galicia. Sala de lo ContenciosoAdministrativo. Sentencia n. 2969/2013. Id Cendoj: 15030330022013100283. A Coruña, 12 de abril de 2013. [on line] Disponible en: http://www.poderjudicial.es/search/doAction?action=contentpdf\&databasematch=AN\& reference $=6741217$ \&links $=$ \&optimize $=20130611$ \&publicinterface=true $]$ [Fecha de consulta: 1 sep. 2014]

Real Decreto 1015/2009, de 19 de junio. Regula la disponibilidad de medicamentos en situaciones especiales. Boletín Oficial del Estado, Madrid, 20 de julio de 2009. [on line] Disponible en http://www.boe.es/diario boe/txt.php?id=BOE-A2009-12002 [Fecha de consulta: 1 sep. 2014]

Ley 29/2006, de 26 de julio. Garantías y Uso Racional de los Medicamentos y Productos Sanitarios. Boletín Oficial del Estado, Madrid, 27 de julio de 2006. [on line] Disponible en http://www.boe.es/buscar/doc.php?id=BOE-A-2006-13554ec [Fecha de consulta: 1 septiembre 2014]

ESTEVE PARDO, José. El desconcierto del Leviatán política y derecho ante las incertidumbres de la ciencia. Madrid: Marcial Pons, 2009. 211 p. ISBN: 9788497685856.

EURORDIS. Rare Diseases Europe. [on line] Disponible en www.eurordis.org/es [Fecha de consulta: 1 sep. 2014]

FARMAMUNDI. Cooperación al desarrollo y medicamentos esenciales. Conclusiones del II Curso de Salud y Atención Farmacéutica en Países en Vías de Desarrollo (2427 de abril del 2001). Alfafar (Valencia), 2002. 218 p. ISBN: 8493249602.

FEDER. Federación Española de Enfermedades Raras. [on line] Disponible en www.enfermedades-raras.org [Fecha de consulta: 1 sep. 2014]

FICICCHIA, Federica. Malattie rare e farmaci orfani. Profili giuridici. Contratto e impresa. Europa,Torino, (1):428-454, 2007. ISSN: 11272872. 
GARRIDO CUENCA, Nuria. Hacia una construcción del derecho a la salud como contenido del derecho fundamental a una vida digna y de calidad: el "mínimo vital" como límite de la sostenibilidad económica. En: BALAGUER CALLEJÓN, Francisco y ARANA GARCÍA, Estanislao. Libro homenaje al profesor Rafael Barranco Vela. Cizur Menor (Navarra): Thomson Reuters-Civitas, 2014. 3208 p. ISBN: 9788447049080.

La estrategia europea de enfermedades raras: Desafíos y oportunidades de una acción sostenible global. En: PÉREZ GÁLVEZ, Juan Francisco y BARRANCO VELA, Rafael (Dirs.). Derecho y salud en la Unión Europea. Granada: Comares, 2013. 376 p. ISBN: 9788490450710.

IIER. Instituto de Investigación de Enfermedades Raras. [on line] Disponible en http://www.isciii.es [Fecha de consulta: 1 sep. 2014]

LARIOS RISCO, David, PALOMAR OLMEDA, Alberto y VÁZQUEZ GARRANZO, Javier. La reforma sanitaria. Cizur Menor (Navarra): Thomson-Aranzadi, 2012. 384 p. ISBN: 9788490141922.

MORENO TAPIA, Irene y SABATER, Julio. La autorización condicional de medicamentos en la Unión Europea. Cuadernos de Derecho Farmacéutico, Madrid, (17):21-24, 2006. ISSN: 15795926.

NÚÑEZ LOZANO, $M^{\underline{a}}$ Carmen. Régimen jurídico del uso compasivo de medicamentos. Revista Española de Derecho Administrativo, Madrid, (130):327-346, 2006. ISSN: 0210-8461.

El régimen jurídico del uso compasivo y del uso alternativo de medicamentos. Revista de Administración Pública, Madrid, (184):273-305, 2011. ISSN: 00347639.

PANUNZIO, Sergio y RECCHIA, Giorgio (Dirs.): Malattie rare: la ricerca tra etica e diritto. (Atti del convegno di studi, Roma, 14 febbraio 2006). Giuffré: Milano, 2007. 233 p. ISBN: 8814134456.

SALVATORE, Vincenzo (Dir.). Le Agenzie dell'Unione Europea. Profili istituzionali e tendenze evolutive. Pavia: Polo Interregionale di Eccellenza Jean Monnet, 2011. 228 p. ISBN: 9788896890028.

UNIÓN EUROPEA. Reglamento (CE) 141/2000, del Parlamento Europeo y del Consejo, de 16 de diciembre de 1999, sobre medicamentos huérfanos. Diario Oficial de la Unión Europea, Bruselas, 22 de enero de 2000. [on line] Disponible en http://eur-lex.europa.eu/legal-

content/ES/TXT/PDF/?uri=CELEX:32000R0141\&from=ES [Fecha de consulta: 1 sep. 2014]

Reglamento (CE) 507/2006, de la Comisión, de 29 de marzo de 2006, sobre la autorización condicional de comercialización de los medicamentos de uso humano que entran en el ámbito de aplicación del Reglamento (CE) 726/2004 del Parlamento Europeo y del Consejo. Diario Oficial de la Unión Europea, Bruselas, 30 de marzo de 2006. [on line] Disponible en http://ec.europa.eu/health/files/eudralex/vol1/reg 2006 507/reg 2006507 es.pdf [Fecha de consulta: 1 sep. 2014]

VALVERDE LÓPEZ, José Luis. La especificidad del Estatuto Jurídico del Medicamento. En: BALAGUER CALLEJÓN, Francisco y ARANA GARCÍA, Estanislao. Libro homenaje al profesor Rafael Barranco Vela. Cizur Menor (Navarra): Thomson

Reuters-Civitas, 2014. 3208 p. ISBN: 9788447049080. 
Los medicamentos genéricos en el marco jurídico de la Unión Europea. En: LÓPEZ GUZMÁN, José. Medicamentos genéricos: una aproximación interdisciplinar. Pamplona: EUNSA, 2007. 270 p. ISBN: 8431324708.

VIDA FERNÁNDEZ, José. La prestación farmacéutica. En: MONEREO PÉREZ, José Luis, MOLINA NAVARRETE, Cristóbal y MORENO VIDA, Ma Nieves (Dirs.). Comentario a la legislación reguladora de la sanidad en España. Régimen jurídico de la organización sanitaria, personal sanitario y prestaciones sanitarias. Granada: Comares, 2007. 560 p. ISBN: 9788498362541.

VÍRGALA FORURIA, Eduardo. Las agencias reguladoras de la Unión Europea. Granada: Comares, 2011. 179 p. ISBN: 9788498368949.

Recebido para publicação em 26 de agosto de 2014. Admitido para publicação em 1ำ de dezembro de 2014. 